\title{
ESTRUTURA DIAMÉTRICA E ESPACIAL DE PIQUIAZEIRO NA COMUNIDADE DE PIQUIATUBA, FLORESTA NACIONAL DO TAPAJÓS, OESTE DO PARÁ
}

\author{
Mauriceia Colares Lima'; Sheyla Regina Marques Couceiro; Élcio Meira da Fonseca Júnior³. \\ ${ }^{1}$ Universidade Federal do Oeste do Pará, Santarém, Pará, Brasil, mlcolares@outlook.com \\ 2 Universidade Federal do Oeste do Pará, Santarém, Pará, Brasil, sheylacouceiro@yahoo.com.br \\ ${ }^{3}$ Universidade Federal do Oeste do Pará, Santarém, Pará, Brasil, meirafonseca@yahoo.com.br
}

RESUMO: Caryocar villosum (Aubl.) Pers. (Caryocaraceae) é uma árvore de grande porte, nativa da região amazônica, popularmente conhecida como Piquiá ou Piquiazeiro. Sua madeira e fruto são bem conhecidos e utilizados na região, onde geralmente é coletada de modo extrativista. O presente estudo teve como objetivo determinar a densidade, distribuição espacial e diamétrica da população de C. villosum em duas áreas de 25 ha cada, localizadas na comunidade de Piquiatuba, Floresta Nacional do Tapajós (Flona Tapajós), no Oeste do Pará, sendo uma área de floresta secundária com maior exploração dos frutos e a outra área de floresta primária preservada. Foram medidos os diâmetros das árvores a 1,30 m do solo, a altura das árvores e a distância entre árvore. Na área de floresta secundária observouse 11 indivíduos com densidade de 0,44 indivíduos por hectare. Na área de floresta primária registrou-se apenas quatro indivíduos com densidade de 0,16 indivíduos por hectare. Em ambas as áreas houve distribuição agregada, com predominância de indivíduos adultos (produtores de frutos), com altura média de 27,60 m ( \pm 7,28 m) na área de floresta secundária e 32,08 m $( \pm 5,12 \mathrm{~m})$ na área de floresta primária. A estrutura diamétrica da população apresenta lacunas de indivíduos com baixo diâmetro, sugerindo dificuldade de recrutamento. Isto indica a necessidade de estudos sobre germinação, dispersão de sementes e o desenvolvimento de mudas para restaurar áreas de exploração na região ou tratamentos silviculturais que favoreçam a regeneração natural da espécie na comunidade de Piquiatuba.

Palavra-chave: Amazônia; espécie florestal; ecologia; importância econômica.

\section{DIAMETRIC AND SPATIAL STRUCTURE OF PIQUIAZEIRO IN THE COMMUNITY OF PIQUIATUBA, TAPAJÓS NATIONAL FOREST, WEST OF PARÁ}

ABSTRACT: Caryocar villosum (Aubl.) Pers. (Caryocaraceae) is a large tree, native to the Amazon region, popularly known as Piquiá or Piquiazeiro. Its wood and fruit are well known and used in the region, where it is generally collected in an extractive way. 
The present study aimed to determine the density, spatial and diametric distribution of the C. villosum population in two areas of 25 ha each, in the community of Piquiatuba, located in the Tapajós National Forest (FLONA Tapajós), West of Pará, being an area of secondary forest with greater fruit exploitation and the other area of preserved primary forest. The tree diameters were measured at $1.30 \mathrm{~m}$ from the soil, the height of the trees and the distance between tree. In the secondary forest area, 11 individuals with a density of 0.44 individuals per hectare were observed. In the primary forest area only four individuals with density of 0.16 ha individuals were recorded. In both areas there was an aggregate distribution, with predominance of adult individuals (fruit producers), with mean height of $27.60 \mathrm{~m}( \pm 7.28 \mathrm{~m})$ in the secondary forest area and $32.08 \mathrm{~m}( \pm 5.12 \mathrm{~m})$ in the primary forest area. The diametric structure of the population presents gaps in low-diameter individuals, suggesting difficulty in recruiting. That indicates the need for studies on germination, seed dispersal and seedling development to restore areas of exploitation in the Piquiatuba community.

KEYWORDS: Amazonia, Ecology, Forest species.

\section{ESTRUCTURA DIAMÉTRICA Y ESPACIAL DE PIQUIAZEIRO EN LA COMUNIDAD DE PIQUIATUBA, BOSQUE NACIONAL DEL TAPAJÓS, OESTE DE PARÁ}

Resumen: Caryocar villosum (Aubl.) Pers. (Caryocaraceae) es un árbol de gran porte, nativo de la región amazónica, popularmente conocido como Piquiá o Piquiazeiro. Su madera y fruto son bien conocidos y utilizados en la región, donde generalmente es recolectado de modo extractivo. El presente estudio tuvo como objetivo determinar la densidad, distribución espacial y distribución diamétrica de la población de C. villosum en dos áreas de 25 ha cada uno, ubicadas en la comunidad de Piquiatuba, Bosque Nacional del Tapajós (Flona Tapajós), en el Oeste de Pará, siendo una de ella situada en un bosque secundario con mayor explotación de frutos y la otra en bosque primario preservado. Se midieron el diámetro de los árboles a 1,30 m del suelo, la altura de los árboles y la distancia entre árboles. En el área de bosque secundario se observaron 11 individuos con una densidad de 0,44 individuos por hectárea. En el área de bosque primario se registraron sólo cuatro individuos con una densidad de 0,16 individuos por ha. En ambas áreas hubo distribución agregada con predominio de individuos adultos (productores de frutos), con una altura media de 27,60 m $( \pm 7,28$ $\mathrm{m}$ ) en el área de bosque secundario y 32,08 m ( $\pm 5,12 \mathrm{~m}$ ) en el área de bosque primario. La estructura diamétrica de la población presenta ausencia de individuos con bajo diámetro, lo que sugiere la dificultad de reclutamiento. Esto indica la necesidad de realizar estudios sobre la germinación, la dispersión de semillas y el 
desarrollo de los renovales para restaurar las áreas de explotación en la comunidad de Piquiatuba.

PALABRAS CLAVE: Amazonia, Ecología, Especies forestales.

\section{INTRODUÇÃO}

A floresta amazônica é a maior floresta tropical do mundo (BARROSO et al., 2012), ocupando quase metade do território brasileiro (OLIVEIRA et al., 2008), abrigando uma rica biodiversidade, constantemente ameaçada pelas atividades humanas em muito associadas ao desmatamento e exploração de madeira (e.g. WEAM et al., 2012).

Na tentativa de se conservar parte desse patrimônio mundial foram concebidas algumas Unidades de Conservação (UC), entre as quais a Floresta Nacional do Tapajós (Flona Tapajós), criada pelo decreto no. 73.684 de 19 de fevereiro de 1974, abrangendo uma área de 544.927 hectares entre os municípios de Aveiro, Belterra, Placas e Rurópolis, no Oeste do Pará. A Flona Tapajós permite a exploração múltipla dos recursos naturais de forma sustentável por seus comunitários, o que inclui atividades extrativistas e exploração de madeira (IBAMA, 2004).

Entre as espécies vegetais de grande importância socioeconômica presentes na Flona Tapajós encontra-se a Caryocar villosum (Aubl.) Pers. (Caryocaraceae), popularmente conhecida como piquiá ou piquiazeiro. C. villosum é uma árvore de grande porte ( $50 \mathrm{~m}$ de altura e 2,5 m de diâmetro; CARVALHO et al., 2006). Sua madeira é de grande qualidade (MARX et al., 1997; MARTINS; GRIBEL, 2007), serve para fabricação de objetos, móveis e construção de casas (COSTA; MITJA, 2010). Assim, até o final dos anos 90, o piquiá estava entre as 16 espécies madeireiras comercializadas internacionalmente pelo polo Santarém-PA (IBAMA, 2011). Hoje encontra-se ameaçada de extinção 
(SILVA, 2013).

C. villosum também fornece produtos de interesse ao mercado de produtos florestais não madeireiros (CORRÊA et al., 2015), pois seus frutos são matéria-prima para produção de óleo, azeite, licor e sabão comercializados regionalmente (CARVALHO et al., 2006; CHISTÉ et al., 2012). Suas amêndoas também podem ser uma excelente fonte de alimentação para as pessoas e seu óleo pode ser utilizado na indústria cosmética, farmacêutica, alimentar e energética (SHANLEY; MEDINA, 2005).

Para a comunidade de Piquiatuba, localizada na Flona Tapajós, no município de Belterra-PA, C. villosum é uma espécie muito importante pois contribui na renda dos comunitários (GALUPPO, 2004). O óleo de piquiá extraído do fruto é diretamente comercializado pelos produtores no mercado de Santarém-PA, principalmente, para o uso medicinal (GONÇALVES et al., 2012). Além destes,

a Cooperativa Mista da Flona Tapajós (COOMFLONA) também é responsável pela venda até o consumidor final ou pela negociação para exportação como matéria-prima para as empresas farmacêuticas internacionais (GONÇALVES et al., 2012).

Porém é importante ressaltar que $C$. villosum apresenta dificuldades de propagação, comumente efetuado por sementes, sendo a germinação lenta, baixa e desuniforme (VILLACHICA et al., 1996), especialmente pela exigência de luminosidade no desenvolvimento inicial dos espécimes (SILVA, 2013).

A superexploração da espécie sem estudos ecológicos prévios na região, associada às crescentes modificações do ambiente seja pelos comunitários seja pelas madeiras que operam na região, podem comprometer populações locais de C. villosum. Assim, o objetivo desse trabalho foi determinar a densidade, distribuição espacial e diamétrica da população de C. villosum, comparando duas áreas na comunidade de Piquiatuba, que explora a espécie, na Floresta Nacional do Tapajós. Essas informações podem ser úteis para propor planos de manejo 
e conservação desta unidade.

\section{MATERIAL E MÉTODOS}

A Floresta Nacional do Tapajós está localizada no Oeste paraense, entre os paralelos de $2^{\circ} 4^{\prime}$ e $4^{01} 0^{\prime}$ de latitude Sul e entre os meridianos de 54045' e 55030'de longitude Oeste, abrangendo os municípios de Belterra, Aveiro, Placas e Rurópolis, com área estimada em 544.927 hectares (ESPÍRITO-SANTO et al., 2005). A comunidade de Piquiatuba, abrange 2.153 hectares da Flona, sendo localizada na margem direita do rio Tapajós, município de Belterra (IBAMA, 2004) (Figura 1), entre as comunidades de Pedreira e Marituba.

A Flona Tapajós (Figura 1) é caracterizada por uma vegetação de Floresta Ombrófila Densa (IBAMA, 2004). O clima na região é classificado como Af onde a temperatura média anual é superior a $26^{\circ} \mathrm{C}$. A precipitação média anual está entre 1.900 e 2.400 $\mathrm{mm}$, com regime de chuvas apresentando grande variação durante o ano, com as maiores precipitações ocorrendo nos meses de janeiro a maio (ALVARES et al., 2014).

Para amostragem da população de C. villosum foram utilizadas duas áreas de 25 ha com 25 parcelas $100 \times 100 \mathrm{~m}$ cada, sendo uma área de floresta secundária $\quad\left(502^{\circ} 59^{\prime} 26,6^{\prime \prime}\right.$ e W055 $\left.05^{\prime} 42,9^{\prime \prime}\right)$, que apresenta resquícios de extração de madeira e atualmente ocorre extrativismo de frutos de C. villosum e de outras espécies frutíferas como o uxizeiro (Endopleura uchi Huber), sendo mais próximo aos comunitários de Piquiatuba em relação a segunda área. A segunda área é de floresta primária $\left(S 03^{\circ} 00^{\prime} 28,4^{\prime \prime}\right.$ e W055 $\left.02^{\prime} 38,1^{\prime \prime}\right)$, sem uso extrativista, onde se observa a predominância de árvores de grande porte com dossel mais fechado, se comparado com a primeira área.

Em cada área foram amostrados todos os indivíduos de C. villosum, sendo medidos a altura das árvores, o diâmetro altura do peito (DAP, a 1,30 m do solo) e a distância entre árvores. As medições do DAP e da distância entre árvores foram realizadas utilizando 
uma trena de $100 \mathrm{~m}$. A altura total de cada indivíduo foi estimada pelo comprimento de uma linha de náilon presa a um projetil lançado por estilingue a copa da árvore.

Figura 1. Localização da Floresta Nacional do Tapajós. Área de floresta secundária, com extrativismo de frutos de Caryocar villosum (1) e área de floresta primária (2). Fonte: Google Inc.

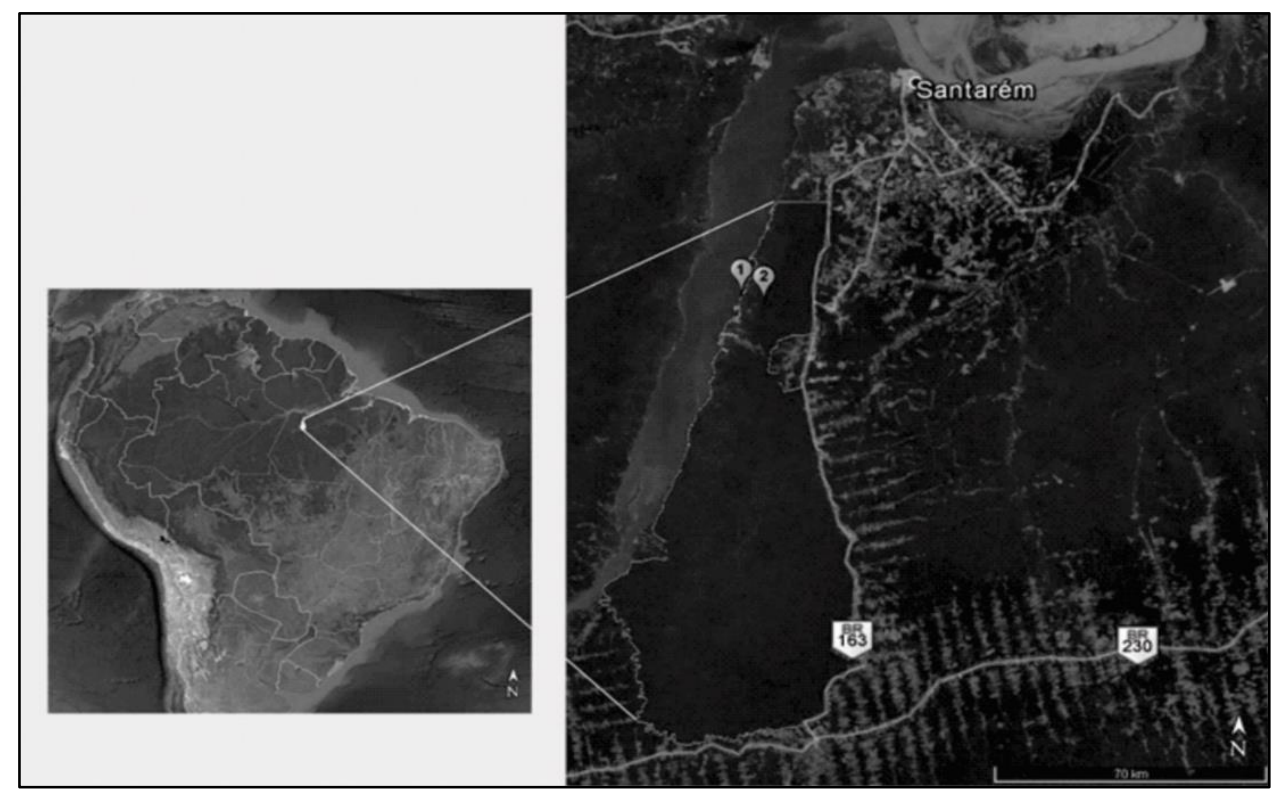

Sob a copa de cada indivíduo de C. villosum foi medida a intensidade luminosa com auxílio de um luxímetro digital SKILL-TEC modelo SKTHAL-01, no horário entre 10:00 às 11:00 h.

A densidade de indivíduos foi calculada pelo número total de indivíduos divididos pela extensão total de cada área. Para análise da distribuição de altura e diamétrica de $C$. villosum foram elaborados gráficos de distribuição de altura e DAP (MARTINS, 2008) por área.

Para análise da distribuição espacial foi utilizado o índice de Morisita (IM) (MORISITA, 1959), onde o valor calculado do $x^{2}$ pode então ser comparado com o valor crítico 
apropriado com ( $\mathrm{n}-1)$ graus de liberdade e um nível de significância a 0,05. Se o índice de Morisita não diferir significativamente de 1, o padrão de distribuição de espécie é aleatório e, se o valor calculado for maior que o valor crítico, o padrão de distribuição é agregado ou uniforme.

\section{RESULTADOS E DISCUSSÃO}

Foi contabilizado um total de 15 indivíduos de C. villosum nas duas áreas, sendo 11 árvores adultas na área de floresta secundária (P1) e, quatro árvores adultas na área de floresta primária (P2).

$\mathrm{Na}$ área de floresta secundária a incidência luminosa variou de 200 a 7.410 lux., com densidade de 0,44 indivíduos por hectare. Os indivíduos apresentaram altura média de 27,60 m ( \pm 7,28 m) e DAP médio de $116,20 \mathrm{~cm}$ $( \pm 70,80 \mathrm{~cm})$.

A maior parte dos indivíduos apresentaram altura e DAP superiores a 24,50 m e 12,70 cm, respectivamente (Figura 2). A elevada frequência de indivíduos de maior porte demostra que o padrão de "J-invertido", com representantes em todas as classes de tamanho e em maior número nas classes menores não foi observado nesta área.

Figura 2. Distribuiç̧ão da população de C. villosum com base em histogramas de altura e de DAP, na área de floresta secundária, na comunidade de Piquiatuba, Flona Tapajós, PA.
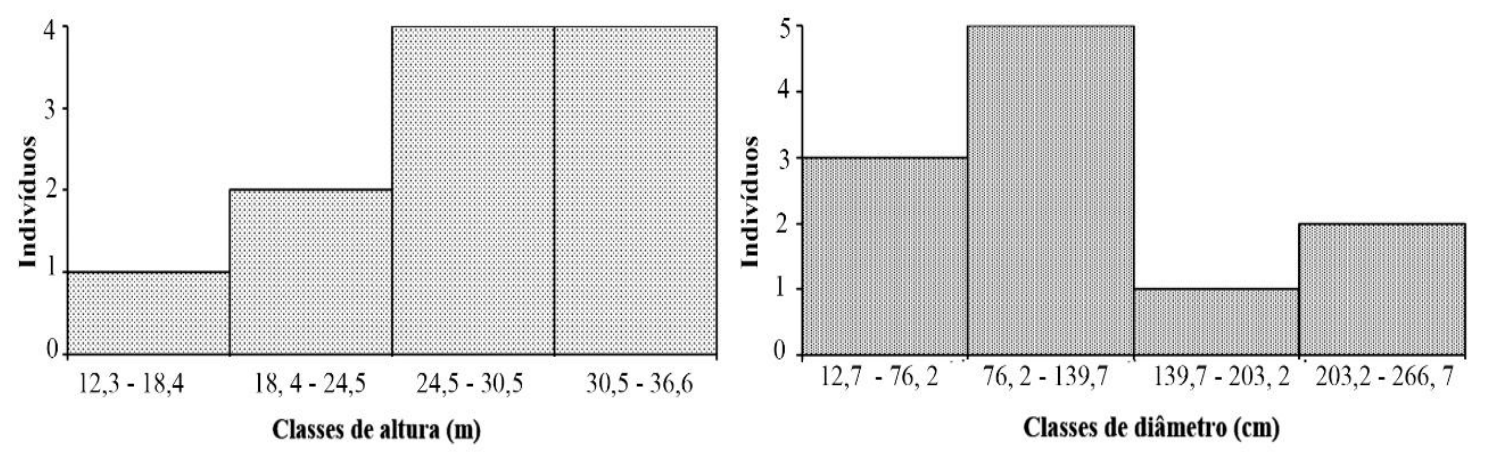
O número máximo de indivíduos em uma única parcela foi igual a dois, sendo que a distância média entre indivíduos, em linha reta, foi de 29,06 m ( \pm 28,73 m). Logo, o padrão de distribuição espacial de indivíduos de C. villosum foi agregado (Figura 3), com índice de Morisita $(\mathrm{IM})=2,27\left(X^{2}\right.$ $=47,73 ; g l=24 ; p<0,05)$.

$\mathrm{Na}$ área de floresta primária, a incidência luminosa variou de 100 a
2.490 lux. Nessa área foram observados quatro indivíduos de C. villosum, correspondendo a uma densidade de 0,16 indivíduos por hectare. Os indivíduos apresentaram altura média de 32,08 m ( \pm 5,12 m) e DAP médio de 132,40 cm ( \pm 49,80 cm). Houve predominância da terceira classe de altura $(33,10$ a 37,00 m) e da primeira classe de DAP $(87,50$ a 165,60 cm) (Figura 4).

Figura 3. Distribuição espacial de C. villosum na área de floresta secundária, na comunidade de Piquiatuba, Flona Tapajós-PA. Distância dos indivíduos na vertical $\left(D_{A(m)}\right)$ e distância na horizontal $\left(D_{B(m)}\right)$.

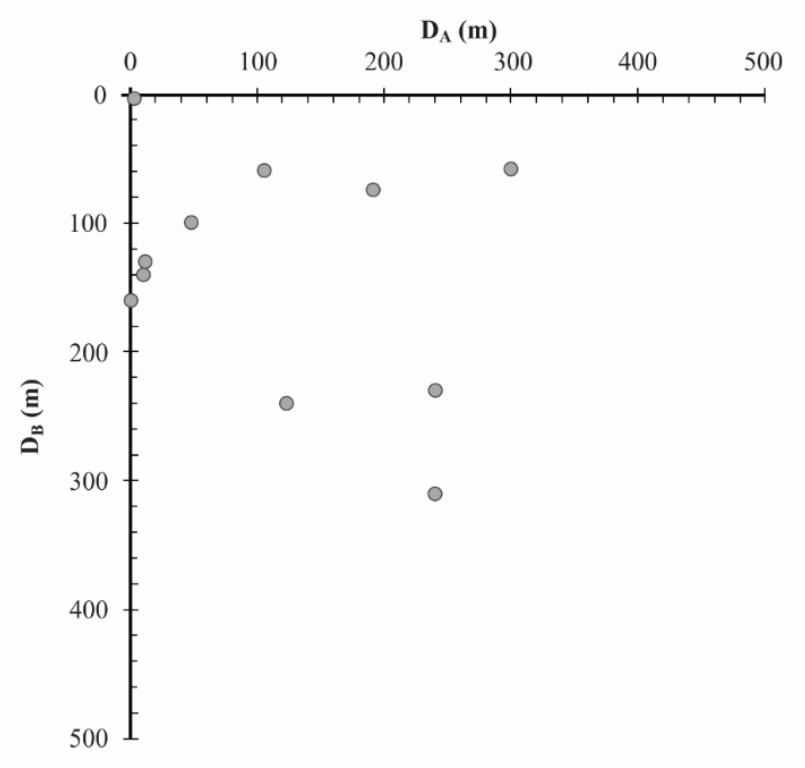


Figura 4. Distribuição da população de C. villosum com base em histogramas de altura e de DAP, na área de floresta primária, na comunidade de Piquiatuba, Flona Tapajós, PA.

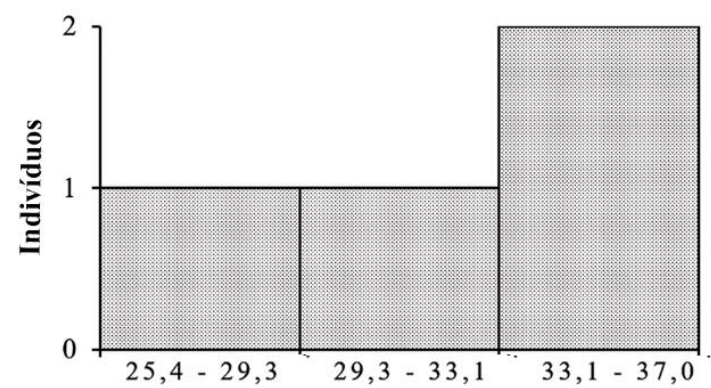

Classes de altura $(\mathrm{m})$

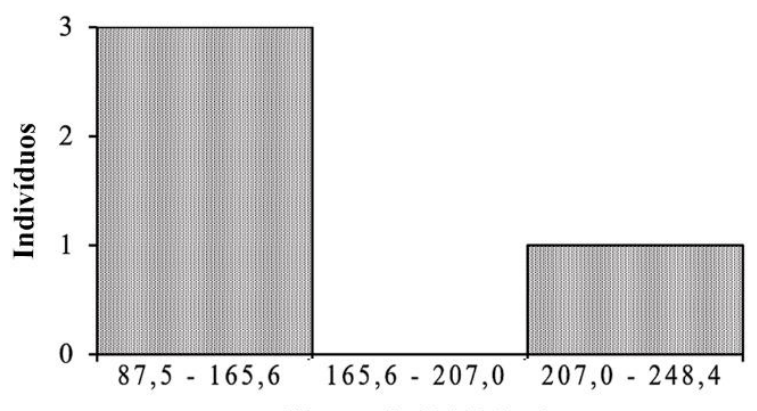

Classes de DAP (cm)
$\mathrm{Na}$ área de floresta primária o número máximo de indivíduos em uma única parcela também foi dois. A distância média entre indivíduos, em linha reta, foi de 155,73 m ( $\pm 192,38 \mathrm{~m})$. Apesar do baixo número de indivíduos observados, o padrão de distribuição espacial de C. villosum nesta área foi também considerado agregado (Figura 5), com valores do Índice de Morisita $(\mathrm{IM})=4.17\left(X^{2}=37,50 ; g l=24 ; p<\right.$ 0,05).

C. villosum é uma espécie de importância econômica e social, seja por sua madeira ou por seus subprodutos. A exploração de C. villosum sem estudos populacionais da espécie contribuem para a diminuição do número de indivíduos, afetando também espécies associadas (SHANLEY; MEDINA， 2005), como morcegos Glossophaginae e Phyllostomidae (Phyllostomus discolor), marsupiais arborícolas e mariposas Sphingidae, definidos como polinizadores mais importantes do piquiá (MARTINS; GRIBEL, 2007).

Na comunidade de Piquiatuba, a distribuição de piquiá apresentou padrão agregado, assim como sua congênere Caryocar brasiliense Camb. (Pequi) apresenta em áreas de Cerrado. Esta distribuição é a mais comum para espécies arbóreas (GUARINO et al., 
2014) e, deve-se a fatores intrínsecos da espécie, tais como reprodutivos e dispersivos ou extrínseco, como características do ambiente (GUARINO et al., 2014). Grandes espécies, como C. villosum precisam de luz para seu desenvolvimento SHANLEY; MEDINA, 2005), e os animais que levam o fruto para comer em clareiras são os maiores responsáveis pela sua propagação (SILVA, 2013).

Figura 5. Distribuição espacial dos indivíduos de C. villosum na área de floresta primária, na comunidade de Piquiatuba, Flona Tapajós, PA. Distância dos indivíduos na vertical $\left(D_{A(m)}\right)$ e distância na horizontal $\left(D_{B(m)}\right)$.

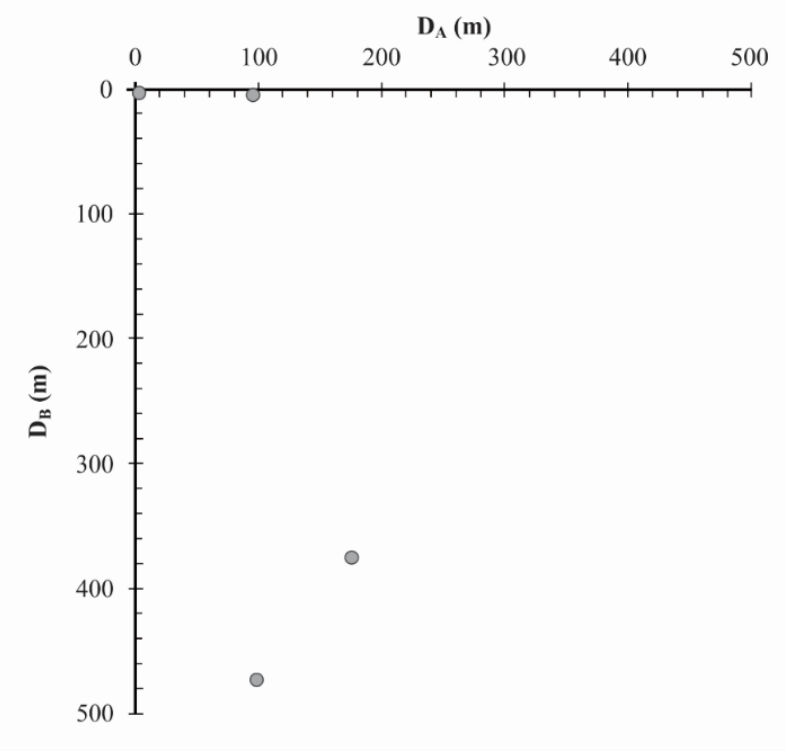

Espécies com distribuição agregada estão entre aquelas com maior probabilidade de extinção local, pois, se as áreas de ocorrência são afetadas por um distúrbio, todos os indivíduos podem não sobreviver (PRIMACK;
RODRIGUES, 2001). Soma-se a isso, o reduzido número de indivíduos com baixo DAP, que em geral são indivíduos jovens para essa espécie, indicando que a população possa estar ameaçada pelo declínio populacional 
pela escassez de recrutamento. Novamente, a derrubada desses poucos indivíduos pode resultar em extinção local pela falta de substitutos (PRIMACK; RODRIGUES, 2001).

A densidade de indivíduos de C. villosum na área de floresta primária foi de 0,16 árvores/ha, o que está muito aquém da ocorrência de C. villosum, de 0,4 a 0,6 indivíduos por hectare observada no estado do Pará (SHANLEY; MEDINA, 2005), sendo que a baixa densidade associada a uma distribuição agregada tendencia à raridade (BRUZINGA et al., 2013).

De acordo com Silva (2013), C. villosum exige muita luminosidade no desenvolvimento inicial, com baixa taxa de germinação do piquiá na floresta. Isso justificaria sua baixa densidade na área de floresta primária, onde observaram-se os indivíduos de maior altura, destacando-se na floresta em busca da luz solar. E esse é um dos motivos de a árvore constar na lista de espécies ameaçadas de extinção (SILVA, 2013).

Apesar da exploração dos frutos exercidos na área de floresta secundária, há maior densidade de C. villosum nessa área em relação à área de floresta primária. O maior número de indivíduos provavelmente esteja relacionado à abertura de pequenas clareiras pela exploração de madeira de outras espécies. Tais clareiras propiciam maior entrada de luz facilitando o estabelecimento e crescimento de um número maior de indivíduos, fato corroborado pela incidência luminosa registrada nas duas áreas, maior na área de floresta secundária. Ou ainda, pela ação humana atuando como dispersor da espécie, durante a coleta e transporte de frutos e sementes. Porém, Gomes et al. (2010), atestam que em clareiras a espécie sofre por ataque de herbívoro diminuindo a taxa de sobrevivência.

\section{CONCLUSÃO}

Os resultados apresentados no momento referem-se à comunidade de Piquiatuba. Devido aos resultados observados de poucos indivíduos, especialmente pela ausência de brotos 
e a escassez de indivíduos de baixo diâmetro (jovens), associada a uma distribuição agregada, indica-se a necessidade de manejo da espécie nesta comunidade, com necessidade de estudos sobre germinação, dispersão de sementes e o desenvolvimento de mudas para restaurar áreas de exploração ou tratamentos silviculturais que favoreçam a regeneração natural da espécie.

Em complemento, visto e extensão do estudo, que foi restrita a uma comunidade da Flona Tapajós, propomos uma amostragem mais ampla para predizer a possibilidade da exploração sustentável dos frutos e produção de óleo de C. villosum nesta Unidade de Conservação como um todo.

\section{AGRADECIMENTOS}

A Universidade Federal do Oeste do Pará pela bolsa de iniciação científica concedida a primeira autora. Ao Instituto Chico Mendes e Conservação da Biodiversidade pela autorização do estudo na Floresta Nacional do Tapajós.

\section{REFERÊNCIAS}

ALVARES, C. A.; STAPE, J. L.; SENTELHAS, P. C.; GONÇALVES, J. L. M.; SPAROVEK, G. Köppen's climate classification map for Brazil. Meteorologische Zeitschrift, Vol. 22, n. 6, p. 711-728, 2014.

BARROSO, M.; COSTA, G.; FERREIRA, M.; VALDUJO, P. ARPA - Biodiversidade. WWF - Brasil. 2012, 34p.

BRUZINGA， J. S.; OLIVEIRA， M. L. R.; MACHADO, E. L. M.; LEITE, H. G.; PEREIRA, I. M.; NOGUEIRA, G. S. Distribuição espacial de indivíduos adultos de pequi. Scientia Forestales, Piracicaba. v. 41, n. 98, p. 249-256, 2013.

CARVALHO, J. E. U.; NASCIMENTO, W. M. O.; MÜLLER, C. H. Propagação do Piquiazeiro. Belém, Embrapa Amazônia Oriental. 2006, 25 p.

COSTA, J. R.; MITJA, D. Uso dos recursos vegetais por agricultores familiares de Manacapuru (AM). Acta Amazonica. v. 40, n. 1, p. 49-58, 2010.

CORREAA, V. V.; GAMA, J. R. V.; RIBEIRO, R. B. S.; ALVES, A. F.; VIEIRA, D. S.; XIMENES, L. C. Estrutura e uso potencial de espécies arbóreas em florestas manejadas, PA Moju, Santarém-Pará. Cerne. v. 21, n. 2, p 293 300, 2015.

CHISTÉ, R. C.; FREITAS, M.; MERCADANTE, A. Z.; FERNANDES, E. 
The potential of extracts of Caryocar villosum pulp to scavenge reactive oxygen and nitrogen species. Food Chemistry, v. 135, n. 3, p.1740-1749, 2012.

GALUPPO, S. C. Documentação do uso e valorização do óleo de piquiá (Caryocar villosum (Aubl) Pers. e do leite do amapá-doce (Brosimum parinarioides) Ducke para a comunidade de Piquiatuba, Floresta Nacional do Tapajós. Estudos físicos, químicos, fitoquímicos e farmacológicos. Belém, 2004. 17 f. Dissertação (Mestrado em Ciências Florestais) - Universidade Federal Rural da Amazônia, Belém, 2004.

GONÇALVES, D. C. M.; GAMA, J. R. V.; OLIVEIRA, F. A. O.; OLIVEIRA JR, R. C.; ARAUJO, G. C.; ALMEIDA, L. S. Aspectos mercadológicos de produtos não madeireiros na economia de Santarém-Pará, Brasil. Floresta e Ambiente. v. 19, n. 1, p. 9-16, 2012.

GOMES, J. M.; CARVALHO, J. O. P.; SILVA, M. G.; NOBRE, D. N. V.; TAFFAREL, M.; FERREIRA， J. E. R.; SANTOS, R. N. J. Sobrevivência de espécies arbóreas plantadas em clareiras causadas pela colheita de madeira em uma floresta de terra firme no município de Paragominas na Amazônia brasileira. Acta Amazonica. v. 40, n. 1, p. 171-178, 2010.

GUARINO, E. S. G; GESSNER, C. M.; WADT, L. H. O.; FONSECA, F. L.; RAPOSO, A. Estrutura diamétrica e espacial de uma população natural de
Carapa guianensis Aubl. (Meliaceae) na Amazônia Sul Ocidental. Scientia Forestales. v. 101, p. 91-99, 2014.

IBAMA - NSTITUTO BRASILEIRO DO MEIO AMBIENTE E DOS RECURSOS NATURAIS E RENOVÁVEIS. Floresta Nacional do Tapajós. Plano de manejo. Informações gerais. IBAMA, 2004. v I. 580p. Brasília.

IBAMA - NSTITUTO BRASILEIRO DO MEIO AMBIENTE E DOS RECURSOS NATURAIS E RENOVÁVEIS. Relação empresa e comunidade no contexto do manejo florestal comunitário e familiar: Uma contribuição do projeto Floresta em Pé. IBAMA/DBFLO, 2011. 318 p. Belém-PA.

MARTINS, G. A. Estatística geral e aplicada. 3.ed, São Paulo: Atlas, 2008. 421p.

MARTINS, R. L.; GRIBEL, R. Polinização de Caryocar villosum (Aubl.) Pers. (Caryocaraceae) uma árvore emergente da Amazônia. Revista Brasil Botânica. v. 30, n. 1, 37-45, 2007.

MARX, F.; ANDRADE, E. H.; A. MAIA, J. $G$. Chemical composition of the fruit pulp of Caryocar villosum. Food Research and Technology. v. 204, 442444, 1997 p.

MORISITA, M. Measuring of the dispersion of individuals and analysis of the distributions patterns. Memoirs of the Faculty of Science. v. 2, 215-235 p. 1959.

OLIVEIRA, M. L.; BACCORO, F. B.; 
BRAGA-NETO, R.; MAGNUSSON, W. E. Reserva Ducke: A biodiversidade amazônica através de uma grade. Attma Disign Editorial, Manaus. 2008, $170 \mathrm{p}$.

SILVA, S. Árvores nativas do Brasil. Ed. Europa. 2013, v. 1, 168 p.

SHANLEY, P.; MEDINA, G. Frutíferas e Plantas Úteis na Vida Amazônica. Em: SHANLEY, P.; GALVÃO, J. Árvores e Cipós - Piquiá (Caryocar villosum (Aubl.) Pres.). Belém: CIFOR, Imazon, 2005, 124-132 p.

PRIMACK, R.B.; RODRIGUES, E. Biologia da Conservação. Ed. Planta. 2001, 328p.

VILLACHICA, H; CARVALHO, J. E. U.; MULLER, C. H.; DIAZ, C. S.; ALMANZA, M. F. Frutales $Y$ Hortalizas Promissórios de La Amazônia. Lima: Tratado de Coooperacion Amazônica. Secretaria Pro-Tempore. 1996, 367p.

WEARN, O. R.; REUMAN, D. C.; EWERS, R. M. Extinction Debt and Winddowns of Conservation Opportunity in the Brazilian Amazon. Science, 337(6091): 228-232. 2012. 